www.jmscr.igmpublication.org Impact Factor 5.84

Index Copernicus Value: 83.27 ISSN (e)-2347-176x ISSN (p) 2455-0450 crossref DOI:_https://dx.doi.org/10.18535/jmscr/v5i4.52

Journal Of Medical Science And Clinical Research

\title{
Colour Doppler Evaluation of Fetomaternal Blood Circulation in Intrauterine Growth Retardation and Perinatal Out Come
}

\author{
Authors \\ T. Sasikala ${ }^{1}$, P.Vydehi $^{2}$ \\ ${ }^{1,2}$ Department of Obstetrics and Gynaecology, Rajiv Gandhi Institute of Medical Sciences Government \\ General Hospital, srikakulam Andhra Pradesh, India
}

\begin{abstract}
Aims and Objectives: The aim of the study is Colour doppler evaluation of fetomaternal blood circulation in intra uterine growth retarted fetuses. Management of the diagnosed 1UGR fetuses in antenatal period. To study the perinatal outcome of the intra uterine growth retarted fetuses, 50 cases in our institution.

Materials and Methods: The present study comprises of Colour Doppler evaluation of diastolic umbilical cord blood flow in clinically suspected IUGR cases and perinatal outcome. In the present study, an attempt is made to assess the velocity changes and spectral waveform changes in umbilical artery and also other -vessels such as uterine artery, fetal middle cerebral artery and fetal dorsal aorta also.The parameters, taken into consideration are SD ratio, resistance index, pulsatile index. Doppler study was started at early weeks of IIIrd trimester and followed up with biweekly ultrasonography and any abnormal USG found appropriate management done.

Results: The total no. of cases in the study are categorised into 4 groups: The No. of cases clinically IUGR, but with normal Doppler 27 cases. The No. of cases clinically IUGR and Doppler abnormal 23 cases. The No. of Doppler positive cases with high risk factors more than 92\%. The No. of Doppler Negative cases with high risk factors $81 \%$.More than $92 \%$ doppler positive cases having high risk factor. More than $50 \%$ of doppler positive cases delivered between 32-34 weeks of gestation. Our study almost consistent with the "Perinatal outcome in doppler positive cases" study done by (Dr. Satish Sharma, C.n. Purandare) FOGSI 1994. In our study doppler positive cases having increased Number of' obstetric intervention.

Conclusion: To conclude Colour Doppler is the best non invasive procedure provided clinically useful information in managing pregnancies with more appropriate decision making and better obstetrical and perinatal outcome.
\end{abstract}

\section{Introduction}

The concept of intrauterine growth retardation was introduced in 1947 by Mac Burney. Battaglia and Lubchenco (1967) ${ }^{(1)}$ defined small for gestational age infants as those whose birth weight were below the 10th percentile for their gestational age (Williams 21st edition) ${ }^{(2)}$. Of all small for gestational age fetuses $25-60 \%$ are appropriate grown, they were small because of constitutional factors (like maternal ethnic group, parity, height and weight. Remaining 1/3rd of cases are true intra uterine growth retarded fetuses. "Intra Uterine growth retardation is the pathologic restriction of the fetal growth pattern resulting in birth weight $<10$ percentile for that gestational age" (Fernando Arias 2nd edition) ${ }^{(3)}$. Depending upon the etiology, period of gestation and the duration of intra uterine insult to the fetal growth, the fetal growth 
varies. $20 \%$ of the IGOR fetuses belongs to Type-I (symmentrical type) IUGR, remaining $80 \%$ belongs to Type-II (asymmentrical type). The IUGR fetuses have more complications during pregnancy, labour and neonatal period. The perinatal mortality reflects the level of overall development in community or country. After introduction of modern diagnostic tools like the USG and colour doppler, the obstetrician could able make a diagnosing IUGR and take appropriate decision for further management. The aim of the study is Colour doppler evaluation of fetomaternal blood circulation in intra uterine growth retarted fetuses. Management of the diagnosed 1UGR fetuses in antenatal period. To study the perinatal outcome of the intra uterine growth retarted fetuses, 50 cases in our institution.

\section{Materials and Methods}

The present study comprises of Colour Doppler evaluation of diastolic umbilical cord blood flow in clinically suspected IUGR cases and perinatal outcome, in Obstetrics and Gynaecology Department, Rajiv Gandh Institute Of Medical Sciences Government General Hospital, srikakulam. The study group includes 50 cases of clinically suspected IGOR cases studied from January 2016 to November 2016. These cases were evaluated in the Department of Radiology, Government General Hospital, srikakulam with Colour Doppler ESAOTE BIOMEDICA AU5 HARMONIC Machine using 3.5MHz transduce;*. The study was approved by the Institutional Human Ethical Committee (IHEC). Informed verbal consent was obtained from all subjects. In the present study, an attempt is made to assess the velocity changes and spectral waveform changes in umbilical artery and also other -vessels such as uterine artery, fetal middle cerebral artery and fetal dorsal aorta also.

The parameters, taken into consideration are SD ratio, resistance index, pulsatile index. Doppler study was started at early weeks of IIIrd trimester and followed up with biweekly ultrasonography and any abnormal USG found appropriate management done. During this period the patient was treated with Low dose aspirin $75 \mathrm{mg}$ daily. $10 \%$ of fructodex solution daily. $(500 \mathrm{ml})$ I.V. followed up with ultrasonography biweekly. Aspirin therapy was stopped when the pregnancy reached 34 weeks of gestation. Termination of pregnancy was done when there is worsening of associated risk factor was found the study group includes age group between 17-35 years, pregnancy with and without medical and obstetrics disorders, third trimester study, clinically suspected IUGR pregnancies with single intrauterine gestation, pregnancies with regular menstrual cycles (3-4/28-30) regular and moderate flow, pregnancies with sure dates. exclusion criteria includes age group lessthan 17,age group more than 35 ,pregnancies less than 26 weeks of gestation, symphisiofundal height with average for gestation age, multiple pregnancies, patients with irregular cycles, prgnencies with anomalous babies.

\section{Results}

Intra uterine growth retarted fetuses have more complications during pregnancy, labour and neonatal period. The small or growth retarded fetus has much greater short term, morbidity and mortality (Brar and Rutherford 1988). ${ }^{(4)}$ Growth failure may permanently affect fetal organs, the consequences of which may be appreciated only later in life. Babies born SGA are physically and developmentally trailing behind their normal counter parts (Pryor 1992). ${ }^{(5)}$ They may have high risk of cerebral palsy (Blair and Stames 1992) (7) Epidemiological study have also found correlation between LBW and subsequent Death from Cardiovascular disease (Barker 1992). ${ }^{(8)}$ The importance of identifying fetal growth retardation is therefore self evident. Perinatal mortality reflects the level of overal development in community or country.

\section{Statistical Analysis}

\section{High Risk Factors for IUGR}

a) Pregnancy induced hypertension.

b) Bad obstetrics history

c) Diabetes mellites. 
d) Chronic Hypertension.

e) Heart disease.

f) Renal disorders like glomerulonephritis.

g) Anemia

h) Combination of two (or) more of the above risk factors.

\section{Age Distribution of Cases}

The highest age group in this study is below 20 years, followed by 21 to 25 years.

\begin{tabular}{|l|l|}
\hline Age in Years & Study Group \\
\hline & \\
$17-20$ & 27 \\
$21-25$ & 18 \\
$26-30$ & 5 \\
$31-35$ & - \\
\hline
\end{tabular}

PIA CHART OF AGE DISTRIBUTION OF CASES IN OUR STUDY

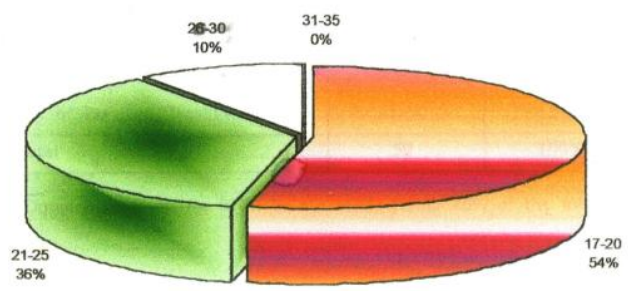

\section{Criteria For Doppler Ositivity}

Systolic and Diastolic Ratio - >3

Spectral waveforms with

a) Reduced end distostolic flow

b) Absent end distostolic flow

c) Reverse end distostolic flow

\section{Percentage of Doppler Positive Cases}

\begin{tabular}{|l|c|c|}
\hline & No. of Cases & Percentage \\
\hline No. of Doppler Positives & $23 / 50$ & 46 \\
No. of Doppler Negatives & $27 / 50$ & 54 \\
\hline Total & 50 & $100 \%$ \\
\hline
\end{tabular}

Doppler positivity for IGGR in our study forms $46 \%$ of the total No.of cases
Umbilical Artery: Significance of AEDV

\begin{tabular}{|l|l|l|}
\hline Parameter & Katherie Wenstrom & Present Study \\
\hline $\begin{array}{l}\text { Mo. of cases with } \\
\text { AEDV in UA }\end{array}$ & $22 / 450-4.9 \%$ & $4 / 23-17.39 \%$ \\
\hline
\end{tabular}

In our study we found that AEDV in umbilical artery is more sensitive in predicting adverse fetal outcome comparable with the study done by Katherine Wenstrom (AEDV- Absent and Diastolic Volume).

\section{Uterine Artery: D1astolic Notch}

\begin{tabular}{|l|c|c|}
\hline & No. of Cases & Percentage \\
\hline $\begin{array}{l}\text { No. of Cases with diastolic } \\
\text { notch }\end{array}$ & 11 & $47.82 \%$ \\
$\begin{array}{l}\text { No. of Cases without } \\
\text { diastolic notch }\end{array}$ & 12 & $52.17 \%$ \\
\hline Total & 23 & $100 \%$ \\
\hline
\end{tabular}

The table shows the no. of cases with diastolic notch is approximating the same without the notch.

\section{Uterine Artery: Percentage Sensitivity}

\begin{tabular}{|l|c|c|}
\hline & No. of Cases & Percentage \\
\hline $\begin{array}{l}\text { No. of Cases with positive } \\
\text { uterine artery doppler } \\
\begin{array}{l}\text { No. of Cases with negative } \\
\text { uterine artery doppler }\end{array}\end{array}$ 10 & $43.47 \%$ \\
\hline Total & 23 & $56.52 \%$ \\
\hline
\end{tabular}

The above table shows the sensitivity of uterine artery doppler study is above $43 \%$.

\section{Middle Cerebral Artery: Sensitivity}

\begin{tabular}{|l|l|l|}
\hline & $\begin{array}{l}\text { No. } \\
\text { Cases }\end{array}$ & of \\
\hline $\begin{array}{l}\text { No. of Cases with } \\
\text { abnormal MCA Doppler }\end{array}$ & 9 & $39.15 \%$ \\
$\begin{array}{l}\text { No. of Cases with } \\
\text { normal MCA Doppler }\end{array}$ & 14 & $60.86 \%$ \\
\hline Total & 23 & $100 \%$ \\
\hline
\end{tabular}

Nearly $39 \%$ of cases showed abnormal MCA Doppler findings in our study. 


\section{JMSCR Vol||05||Issue||04||Page 20046-20053||April}

\section{IUGR: Clinical Vs Doppler Study Sensitivity}

\begin{tabular}{|l|c|c|}
\hline & No. of Cases & Percentage \\
\hline $\begin{array}{l}\text { No. of Cases with clinical } \\
\text { IUGR but Doppler normal }\end{array}$ & 27 & $54 \%$ \\
$\begin{array}{l}\text { No. of Cases with clinical } \\
\text { IUGR but Doppler abnormal }\end{array}$ & 23 & $46 \%$ \\
\hline Total & 50 & $100 \%$ \\
\hline
\end{tabular}

The above table shows that $<50 \%$ of cases having abnormal doppler.

\section{Perinatal Outcome in Doppler Positive Cases}

\begin{tabular}{|l|c|c|}
\hline & $\begin{array}{c}\text { No. of } \\
\text { Cases }\end{array}$ & Percentage \\
\hline Still birth & 4 & $18.75 \%$ \\
Fetal distress during labour & 11 & $49.82 \%$. \\
Meconium Staining & 8 & $34.78 \%$ \\
APGAR Score < 7 at 5min. & 10 & $43.47 \%$ \\
NICQ admission & 11 & $49.82 \%$ \\
Neonatal deaths & 2 & $8.69 \%$ \\
\hline Total & & \\
\hline
\end{tabular}

The above table shows that more than $92 \%$ of cases showed adverse fetal outcome.

\section{Fetal Outcome in Doppler Negative Cases}

\begin{tabular}{|c|c|c|}
\hline & $\begin{array}{ll}\text { No. } & \text { of } \\
\text { Cases } & \end{array}$ & Percentage \\
\hline $\begin{array}{lll}\text { Still birth } & \\
\text { Fetal } \quad \text { distress } & \text { during } \\
\text { labour } & & \end{array}$ & $\begin{array}{l}1 \\
8\end{array}$ & $\begin{array}{l}3.70 \% \\
29.62 \%\end{array}$ \\
\hline Meconium Staining & 7 & $25.92 \%$ \\
\hline $\begin{array}{l}\text { APGAR Score < } \\
\text { a't } 5 \mathrm{~min} \text {. }\end{array}$ & 9 & $33.33 \%$ \\
\hline Neonatal deaths & 2 & $7.40 \%$ \\
\hline Total & & \\
\hline
\end{tabular}

The above table shows that more than $70 \%$ of Doppler negative cases showed adverse perinatal outcome.
BAR CHART OF PERINATAL OUTCOME IN DOPPLER POSITIVE CASES

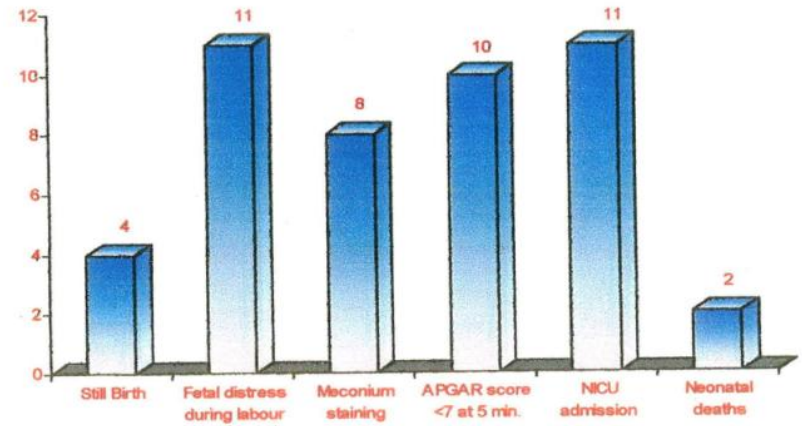

BAR CHART OF FETAL OUTCOME IN DOPPLER NEGATIVE CASES

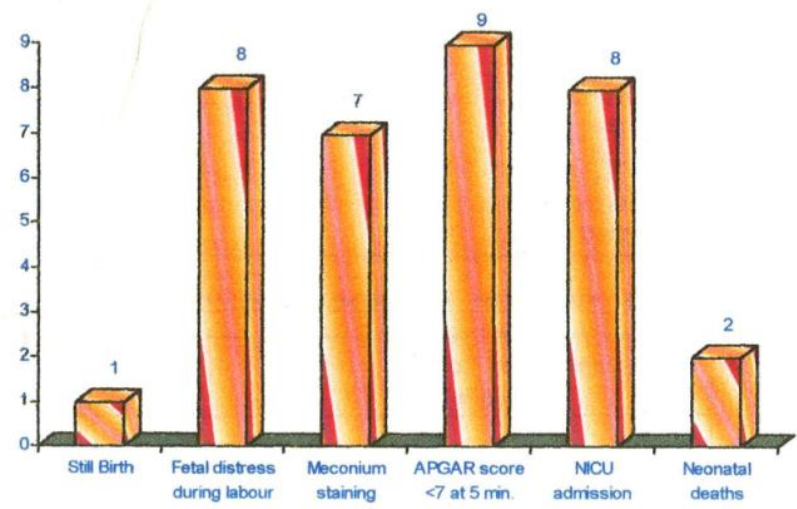

Mode of Deliveries in Doppler Positive Cases

\begin{tabular}{|c|c|c|c|}
\hline 1 & $\begin{array}{l}\text { No. of } \\
\text { Cases }\end{array}$ & Indication & $\begin{array}{c}\text { Percentag } \\
\mathrm{e}\end{array}$ \\
\hline 1. Vaginal Deliveries & & & \\
\hline $\begin{array}{l}\text { a. Normal vaginal } \\
\text { deliveries }\end{array}$ & 7 & & $30.43 \%$ \\
\hline $\begin{array}{ll}\text { b. Abnormal } & \text { vaginal } \\
\text { deliveries } & \text { Forceps }\end{array}$ & 2 & $\begin{array}{l}\text { Prolonged Ilnd } \\
\text { stage with } \\
\text { PIH }\end{array}$ & $8.69 \%$ \\
\hline C. Still birth & 4 & $\begin{array}{l}\text { Cord round } \\
\text { the neck } \\
\text { with } \\
\text { meconium } \\
\text { stained }\end{array}$ & $17.39 \%$ \\
\hline 2. Caesarian Section & 10 & $\begin{array}{c}\text { Fetal } \\
\text { Distress }\end{array}$ & $43.47 \%$ \\
\hline
\end{tabular}


APGAR score in Doppler Positive \& Negative Cases

\begin{tabular}{|c|c|c|c|c|}
\hline Apgar Score & $\begin{array}{l}\text { No. of } \\
\text { Doppler } \\
\text { positive Cases }\end{array}$ & $\mid \begin{array}{l}\text { Percentag } \\
\mathrm{e}\end{array}$ & \begin{tabular}{|l} 
No.of \\
Doppler \\
Negative \\
Cases
\end{tabular} & Percentage \\
\hline $\begin{array}{lll}\text { Less than } & 7 \\
& & \\
\text { at } & 1 & \text { min. }\end{array}$ & 11 & 47.82 & 8 & 29.62 \\
\hline $\begin{array}{l}\text { Less than } 7 \\
\text { at } 5 \text { min. }\end{array}$ & $\begin{array}{l}8 \\
88\end{array}$ & 34.78 & 7 & 25.94 \\
\hline
\end{tabular}

The above table shows that more than $47 \%$ of Doppler positive cases have apgar score $<7$ at 1 minute.

Type of Intra Uterine Growth Retardation in Doppler Positive Cases

\begin{tabular}{|l|c|c|c|}
\hline Symmetric & Percentage & Asymmetric & Percentage \\
\hline 9 & 39.13 & 14 & 60.86 \\
\hline
\end{tabular}

The above table shows that more than $60 \%$ of Doppler positive cases having asymmetrical 1UGR.

\section{Perinatal Outcome in Doppler Positive Cases}

Study done by (Dr. Satish Sharma, C.N. Purandare) FOGSI 1994

\begin{tabular}{|l|c|c|}
\hline & No. of Cases & Percentage \\
\hline Still birth & 3 & 18.75 \\
Fetal distress during labour & 6 & 37.50 \\
Meconium Staining & 9 & 56.25 \\
APGAR Score < at 5min. & 8 & 50.00 \\
NICG admission & 9 & 56.00 \\
Neonatal deaths & 2 & 12.50 \\
\hline
\end{tabular}

The perinatal outcome in doppler positive cases in our study almost consistent with the study done by (Dr. Satish Sharma, C.n. Purandare) (9) FOGSI 1994.
BAR CHART OF PERINATAL OUTCOME IN DOPPLER POSITIVE CASES Study done by (Dr. Satish Sharma, C.n. Purandare) FOGSI 1994

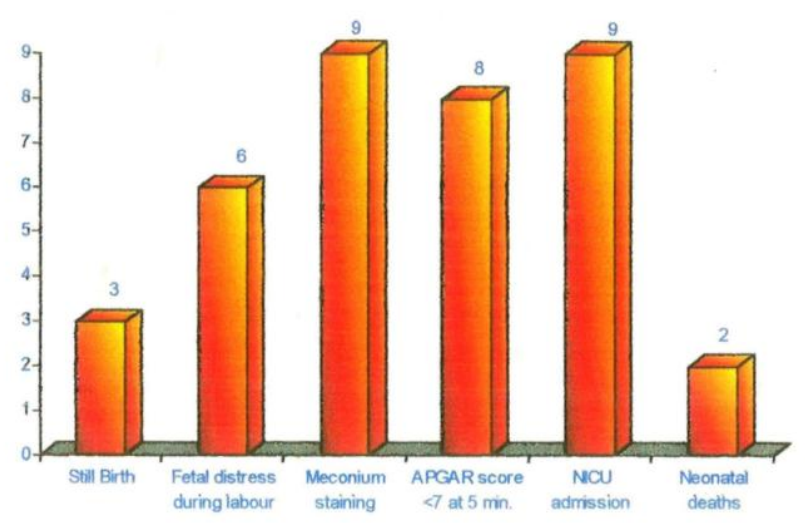

\section{Discussion}

In our present study we have included fifty clinically suspected intrauterine growth retardation cases in third trimester study. The age group in the study was between 17-30 years. None of the Cases are elderly primis.

Of these Cases, 23 Cases (46\%) were found Doppler positive for ICIGR. The remaining 27 cases does not show any evidence of 1QGR on any single Doppler parameter. The Doppler positive cases are followed up with bi-weekly ultrasound examination.

Mean while they were treated with low dose aspirin and oxygen inhalation, high caloric diet, infusion of hypertonic fructodex solution daily. The pregnancy was terminated when there is Worsening of associated risk factor was noted.

\section{Umbilical Artery}

\section{A. S/D Ratio}

The systolic to Diastolic ratio is defined as the ratio of peak systolic velocity to end diastolic velocity. The upper limit for normal value is 3. A systolic to Diastolic ratio of greater than '3' after 30 weeks of gestation is indicative of $1 \mathrm{GGR}$. In our study the maximum no. of Cases (23) showed abnormal S/D ratio ( $>3$ ). Of these Doppler positive Cases 18 cases are having reduced end diastolic flow, and 4 cases having absent end Diastolic flow, 1 Case having -reverse Diastolic flow. The adverse perinatal outcome was more in reverse diastolic flow cases 
than absent diastolic flow cases and reduced diastolic flow cases. The normal value of S/D ratio is 2.6. after 30 weeks of gestation. Increased values are seen with the cases of growth retardation. More than $40 \%$ of cases showed elevated S/D ratio in the present study

B. Resistence Index (RI): The upper limit for the normal value is 0.68 . increased values indicate that there is increased risk of lUGR. In our study lessthan $50 \%$ of cases showed abnormal resistence index.

C. Pulsatile Index (Pi): The normal Pulsatile Index in Uterine Artery is 1. 1. it is increased in cases of IUGR. In the present study the PI of Uterine Artery is increased in More than $50 \%$ of cases.

\section{Uterine Artery Diastolic Notch}

Diastolic Notch: It is defined as a "decrease in the Maximal flow velocity below the maximum diastolic velocities, occurring just after the systolic wave". In non pregnant state the Uterine Artery is a high resistence vessel. Low Diastolic flow and early Diastolic notch is a normal feature of the non pregnant Uterine Circulation. During second trimester the trophoblast invades the Myometrium converting the high resistence flow pattern into that of low resistence pattern, charectarised by increase in diastolic flow and disappearance of the notch.

Persistence of Notch after 26 weeks of gestation is an indicator of PIH or IUGR or both. Persistence of Notch indicates unaltered vasospasm Disappearance

\section{C.Umbilical Artery Resistence Index (RI)}

Resistence Index is given by the formula

$\mathrm{RI}=$

Peak Svstolic velocity - end Diastolic Velocity Peak Systolic velocity

The normal value of RI of umbilical Artery is 0.6. In cases of IUGR it is elevated. In our study more than $2 / 3$ rd of cases show abnormal resistence index.
C. Umbi Lical Artery Pulsati Le Index (PI)

It is calculated by formula $\mathrm{PI}=$

Peak Systolic Velocity- End Diastolic Velocity

Mean velocity

The normal range is 0.4 to I .1. It is increased in cases of growth retardation. This shows that the PI of umbilical Artery is increased in cases with IUGR which is in consistant with the study of John Vugt. The abnormal umbilical Doppler study (SD Ratio) is more sensitive than fetal Doppler which is comparable with the study of JANE. A. BATES. Absent end diastolic velocity and reverse end diastolic velocity is $>$ more sensitive inn predicting adverse fetal outcome comparable with study done by KATHERINE WENSTROM.(10)

\section{Uterine Artery}

A. S/D Ratio: Of the Notch will occur first in the Uterine Artery which is directly under the placenta. In the present study the number of cases with daistolic Notch is approximating the same without the Notch. The number of cases showing Unilateral and Bilateral. Notch forms $43 \%$ Of total number of cases. It is essential to study both the Uterine Arteries because of variation in placentation. In our study if S/D ratio alone is taken into consideration sensitivity of umbilical artery is more than uterine artery. The sensitivity will be maximum if doppler study is done for Uterine and umbilical artery.

\section{Fetal Middle Cerebral Artery}

The mean PI value in Middle Cerebral Artery at 28 weeks of gestation is $1.6 \pm 0.4$ and at 40 weeks it is $1.2 \pm 0.4$. In our study nearly $40 \%$ of cases showed abnormal middle cerebral artery Doppler findings. The PI of umbilical artery is more sensitive than that of other vessels. We found that Abnormal Rl of umbilical artery _ is sensitive in detecting IUGR. 


\section{Fetal Thoracic Aorta \\ Pulsatile Index :}

The normal PI value at 28 weeks of gestation is $2 \pm 0.5$ and at 40 weeks it is about $2 \pm 0.6$. Sensitivity of Aortic Doppler is less than $27 \%$ in our study. So the Doppler study of umbilical artery is more sensitive (46\%) than uterine artery $(43.47 \%)$ than middle cerebral artery $(39.15 \%)$ than thoracic aorta $26.08 \%$.

The total no. of cases in the study are categorised into 4 groups:

1. The No. of cases clinically IUGR, but with normal Doppler 27 cases.

2. The No. of cases clinically IUGR and Doppler abnormal 23 cases.

3. The No. of Doppler positive cases with high risk factors more than $92 \%$.

4. The No. of Doppler Negative cases with high risk factors $81 \%$.

- More than $92 \%$ doppler positive cases having high risk factor.

- More than $50 \%$ of doppler positive cases delivered between 32-34 weeks of gestation.

- Our study almost consistent with the "Perinatal outcome in doppler positive cases" study done by (Dr. Satish Sharma, C.n. Purandare) FOGSI 1994.

- In our study doppler positive cases having increased no.of'obstetric intervention.

\section{Summary}

The objective of every pregnancy is to give birth to a healthy live baby. Umbilical cord, the lifeline of the fetus is the only connecting link between the mother and the fetus, and its normal structure and function is of prime importance for fetal growth and development. Various structural anomalies and pathological process affect umbilical cord, which may result in early fetal death (or) preterm labour (or) growth restriction and increased operative (or) assisted deliveries which leads to increased perinatal morbidity and mortality. So studying the various fetal and maternal vessels by using the colour Doppler during antenatal period and identifying various abnormal determinants will help to implement appropriate treatment modalities. In our study the predictive value to IGGR is more for the umbilical artery (46\%). Of all the Doppler positive cases the maximum number of cases delivery occurred at 32-34 weeks (52.17\%), where as in Doppler negative cases maximum number of cases delivered at 36-37 weeks (37.03\%). So the premature deliveries are more in Doppler positive cases. There is increased incidence of operative deliveries in Doppler positive cases (43.47\%). But in Doppler negative cases operative delivery is less (33.33\%). The APGAR score of $<7$ at $1 \mathrm{mt}$ was $47.82 \%$ in Doppler positive cases and $29.62 \%$ for Doppler Negative cases. In Doppler positive cases the adverse perinatal outcome was observed in $92 \%$, but in Doppler negative cases adverse perinatal outcome observed was $70 \%$ of cases.

\section{Conclusion}

There is a strong correlation between abnormal umbilical artery flow velocity waveform with growth pattern of the fetus and perinatal morbidity and mortality. The umbilical artery flow velocity waveform mainly $\mathrm{S} / \mathrm{D}$ ratio is the more sentitive indicator for identifying the fetuses with intrauterine growth retardation. By studying the flow velocity waveforms of various feto maternal vessels by using the colour Doppler, improves the early detection of intrauterine growth retarded fetuses and initiation of appropriate treatment modalities and thereby reducing the perinatal morbidity and mortality. By studying these various vessels by using the colour Doppler improves the obstetrical decision making, and thereby reducing the perinatal morbidity and mortality. There is increased adverse fetal outcome and obstetric intervention in Doppler positive cases. To conclude Colour Doppler is the best non invasive procedure provided clinically useful information in managing pregnancies with more appropriate decision making and better obstetrical and perinatal outcome.

\section{References}

1. Battaglia fc -> am j obstetrics \& gyneeology 106:1103:1970 
2. Williams: fetal growth disorders 21?t edition page no:743-764. And page no: 1111.

3. Fernando arias: practical guide to high risk pregnancy \& delivery (2nd edition) page no: 301-316.

4. Brar and Rutherford SP:classification of intrauterine growth retardation.semin perinatol 12:2, 1988

5. Pryor JE. Physical and developmental status of preschool small-for-gestational-age children: a comparative study. J Paediatr Child Health. 1992 Apr; 28(2):162-167.

6. Barker DJ. Mothers, babies and health in later life. Edinburgh: Churchill Livingstone, 1998.

7. Blair E, Stanley F. Intrauterine growth and spastic cerebral palsy II. The association with morphology at birth. Early human development. 1992;28(2):91-103.

8. D J Barker, The maternal and fetal origins of cardiovascular disease. J Epidemiol Community Health. 1992 Feb; 46(1): 8-11

9. SATISH SHARMA, C.N.PURANDARE: role of umbilical doppler flow study in high risk pregnancy. (fogsi1994, august: volume 44: no:4, page 518-522).

10. KATHERINE D WENSTROM; Diverse maternal \& fetal pathology associated with absent diastolic flow in the umbilical artery of high risk fetuses- obstet gynaecol (brj): 1991.77: $374=378$. 